\title{
Increased doxorubicin uptake and toxicity in multicellular tumour spheroids treated with DC electrical fields
}

\author{
H Sauer, V Pütz, K Fischer, J Hescheler and M Wartenberg \\ Department of Neurophysiology, University of Cologne, Robert-Koch-Str. 39, D-50931 Cologne, Germany
}

\begin{abstract}
Summary Electrochemotherapy (ECT) is a new approach to the treatment of tumours. In the present study, multicellular prostate tumour spheroids were treated with non-lethal direct current (DC) electrical fields, and uptake and toxicity of doxorubicin were investigated. An electrical field with a field strength of $500 \mathrm{Vm}^{-1}$ applied for a duration of $90 \mathrm{~s}$ resulted in neither reversible nor irreversible membrane breakdown as revealed by fluid phase uptake studies of the membrane impermeant tracer Lucifer yellow. However, treated spheroids showed an increased uptake of doxorubicin and, consequently, an increased toxicity following electrical field exposure. The electrical field raised intracellular reactive oxygen species (ROS) as revealed using $2^{\prime}, 7^{\prime}$-dichlorofluorescein diacetate $\left(\mathrm{H}_{2}\right.$ DCFDA) as an indicator. ROS induced membrane lipid peroxidation since the lipid peroxidation end products malondialdehyde (MDA) and 4-hydroxy-2-(E)-nonenal (4-HNE) were detected after electrical field treatment. Moreover, lipid peroxidation decreased the lipid diffusion coefficient $D$ from $4.2 \times 10^{-10} \mathrm{~cm}^{2} \mathrm{~s}^{-1}$ to $2.7 \times 10^{-10} \mathrm{~cm}^{2} \mathrm{~s}^{-1}$ in the control and treated sample, respectively, as revealed by fluorescence recovery after photobleaching (FRAP) experiments. The field effects could be mimicked by incubating spheroids with $100 \mathrm{~nm}$ hydrogen peroxide and were inhibited by the radical scavengers dehydroascorbate (DHA) and $\alpha$-tocopherol (vitamin $E$ ), indicating that the increased uptake of doxorubicin after electrical field treatment is owing to lipid peroxidation and decreased membrane lipid mobility. Treatment of tumours with low intensity electrical fields may be useful to improve the cytotoxic capacity of anthracyclines.
\end{abstract}

Keywords: electrochemotherapy; electrical field; multicellular tumour spheroid; doxorubicin; lipid peroxidation; lipid diffusion

Electrochemotherapy (ECT) is a promising new approach to fighting cancer and has successfully been applied to regressing tumours in several animal models and clinical trials with squamous cell carcinomas, basal cell carcinomas, metastatic melanomas and liver tumours (Sersa et al, 1995; Glass et al, 1996; Heller et al, 1996; Mir et al, 1997; Ramirez et al, 1998, Belehradek et al, 1993). During ECT treatment, antineoplastic drugs with low cell membrane permeability, e.g. bleomycin (Poddevin et al, 1991), are delivered into cells by short and intense electric field pulses. These field pulses with a field strength of approximately $130000 \mathrm{Vm}^{-1}$ are applied for microseconds and have been discussed to transiently increase the permeability of cell membranes by electroporation (Glass et al, 1996; Nishi et al, 1997). Owing to the high field strength of the applied electrical fields, pulses have to be administered locally at the solid tumour site of avoid serious side-effects of the electrical field on the patient. This implicates that the enhanced delivery of the anticancer agent is restricted to the area that has been electrically treated. Unlocalized tumours, however, remain untreated, making desirable an ECT approach that uses fairly lower field strengths which can be more generally applied to tissues or whole organs.

In the present study, we used multicellular prostate tumour spheroids of the DU-145 cell line to investigate whether treatment with low electrical field strengths increases the uptake and toxicity

Received 11 August 1998

Revised 20 November 1998

Accepted 27 November 1998

Correspondence to: M Wartenberg of the cell membrane-permeant anthracycline doxorubicin. Tumour spheroids were treated for $90 \mathrm{~s}$ with a single electrical field pulse of a field strength that has previously been shown to stimulate tumour cell proliferation (Sauer et al, 1997; Wartenberg et al, 1997). An experimental approach for ECT based on stimulation of cell proliferation should be promising since chemotherapeutics are known to be far more effective in rapid cycling as compared to slow-cycling tumour cells.

\section{MATERIALS AND METHODS}

\section{Culture technique of multicellular tumour spheroids}

The human prostate cancer cell line DU-145 was kindly provided by Dr J Carlsson, Uppsala, Sweden. Cells were cultured in Ham's F10 medium (Gibco, Live Technologies, Helgerman Court, MD, USA) as described previously (Sauer et al, 1997) Spheroids were grown from single cells (passages 2-15) seeded in $250 \mathrm{ml}$ siliconated spinner flasks (Tecnomara, Fernwald, Germany) at $1 \times 10^{5}$ cells $\mathrm{ml}^{-1}$. The spinner flask medium $(175 \mathrm{ml})$ was stirred (20 rpm) using a stirrer system (Tecnomara) and partly changed every day. For the experiments, tumour spheroids of a size class of $150 \pm 30 \mu \mathrm{m}$ were used.

\section{Enzymatic dissociation of multicellular tumour spheroids}

Dissociation of multicellular spheroids was performed as described previously (Wartenberg et al, 1998). In brief, spheroids were washed in phosphate-buffered saline (PBS) and placed in a 


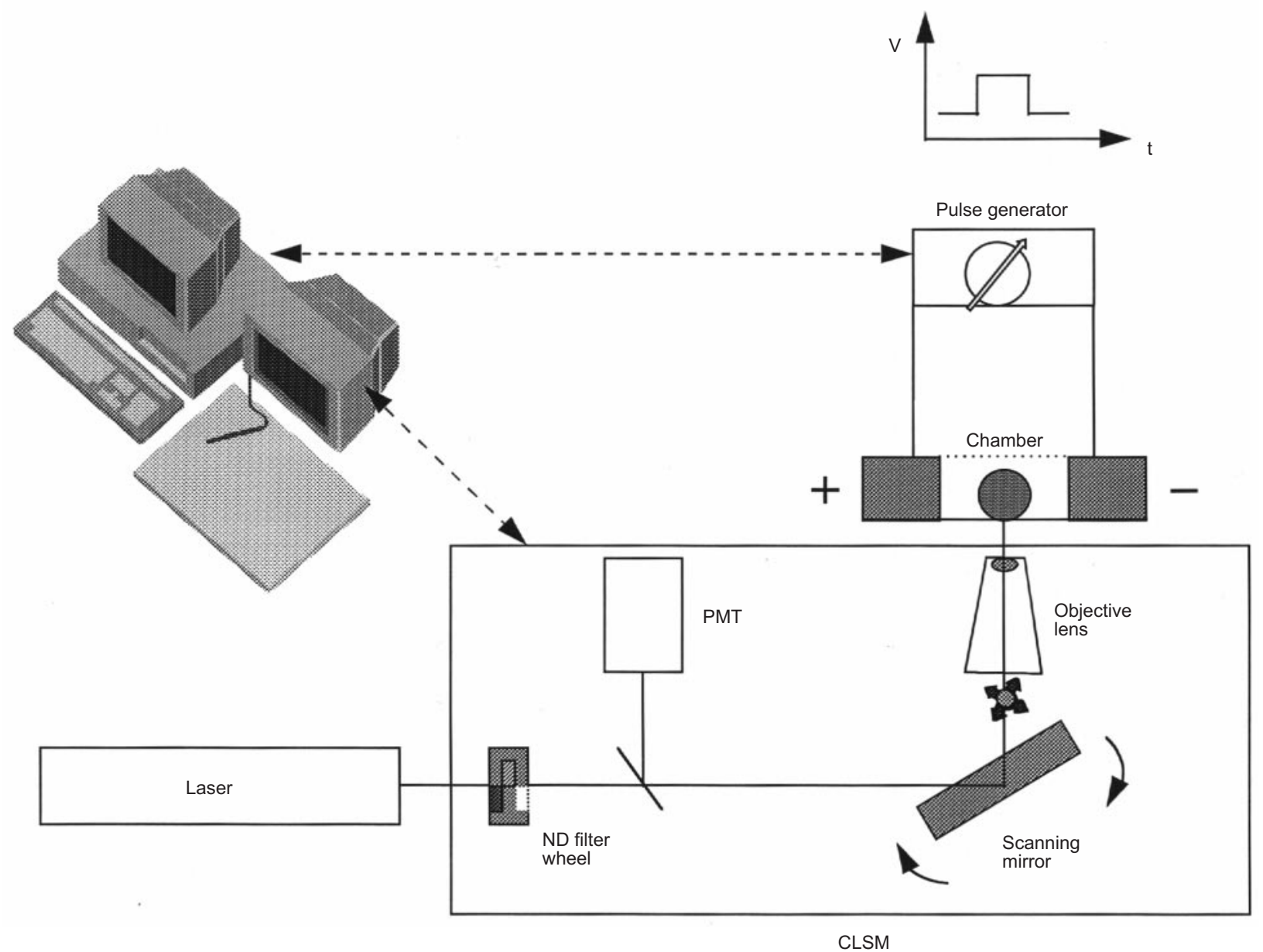

Figure 1 Setup for electrical field treatment of multicellular tumour spheroids. Spheroids were placed into an incubation chamber filled with 'pulsing buffer'. The lateral walls of the chamber were formed by stainless (V4A) steel electrodes with an electrode distance of $2 \mathrm{~mm}$ and an electrode area of $0.4 \mathrm{~cm}^{2}$. A computer-controlled pulse generator generated square wave pulses. During electrical field treatment the spheroids were examined by confocal laser scanning microscopy (CLSM). Fluorescence was recorded by photomultiplier tubes (PMT) and digital images were reconstructed by the CLSM image analysis software. By means of a filter changer (ND filter wheel) the laser beam scanning the tumour spheroids could be attenuated by a factor of approximately 1000 . Flashes for photobleaching experiments could be applied within milliseconds

Petri dish containing $1 \mathrm{ml} 0.2 \%$ Trypsin and $0.05 \%$ ethylenediaminetetraacetic acid (EDTA) in PBS. The spheroids were incubated for $5 \mathrm{~min}$ at $37^{\circ} \mathrm{C}$ and agitated every $30 \mathrm{~s}$. Thereafter they were triturated and the enzymatic reaction was stopped by addition of $4 \mathrm{ml}$ cell culture medium. After centrifugation at $80 \mathrm{~g}$ for $5 \mathrm{~min}$ the cells were resuspended in cell culture medium.

\section{Electrical field treatment}

A schematic scheme of the experimental setup is depicted in Figure 1. For electropulse experiments, multicellular tumour spheroids were suspended in low ionic content, $\mathrm{N}$-2-hydroxyethylpiperazine$\mathrm{N}^{\prime}$-2-ethane-sulphonic acid (HEPES) (5 mM, pH 7.2) buffered 'pulsing medium' (Rols and Teissié, 1990) containing $255 \mathrm{mM}$ sucrose, $1 \mathrm{~mm}$ calcium chloride and $1 \mathrm{~mm}$ magnesium chloride. The pulsing buffer had a conductivity of $500 \mu \mathrm{S} \mathrm{cm}-1$. Spheroids were placed into an incubation chamber between stainless (V4A) steel electrodes, with an electrode distance of $2 \mathrm{~mm}$ and an electrode area of $0.4 \mathrm{~cm}^{2}$. The electrodes were connected to a custommade voltage generator that generated square-wave voltage pulses. One single electric field pulse with a field strength of $500 \mathrm{Vm}^{-1}$ and a duration of $90 \mathrm{~s}$ was applied to the spheroids. The total current in the chamber amounted to $1 \mathrm{~mA}$. The magnetic flux density in the proximity of the multicellular tumour spheroids was calculated to $0.079 \mu \mathrm{T}$, which is below the average laboratory noise level for low frequency electromagnetic fields (Cameron et al, 1993). After electric field treatment tumour spheroids were resuspended in F10 medium and incubated at $37^{\circ} \mathrm{C}$ for $90 \mathrm{~min}$. In control experiments, we ensured that no water electrolysis, $\mathrm{pH}$ or temperature shifts occurred during the electropulse experiments.

\section{Doxorubicin uptake experiments and confocal laser scanning microscopy}

For doxorubicin uptake experiments multicellular tumour spheroids were suspended in pulsing buffer supplemented with $4 \mu \mathrm{M}$ doxorubicin (Sigma, Deisenhofen, Germany). Electropulsing in the presence of doxorubicin was performed under the optical control of an inverted confocal laser scanning microscope (CLSM 410, Carl Zeiss, Jena, Germany) equipped with an immersion corrected, $25 \times$, numerical aperture 0.83 , objective (Plan Neofluar, Carl Zeiss). Images of $512 \times 512$ pixels, 8 bits, were recorded 
A

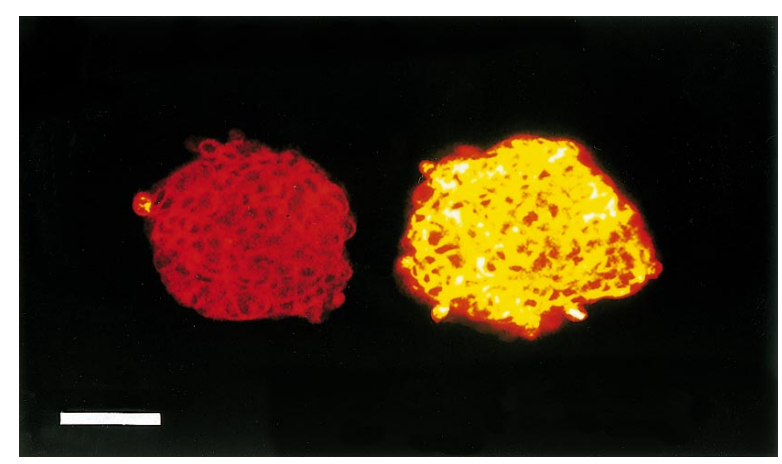

B

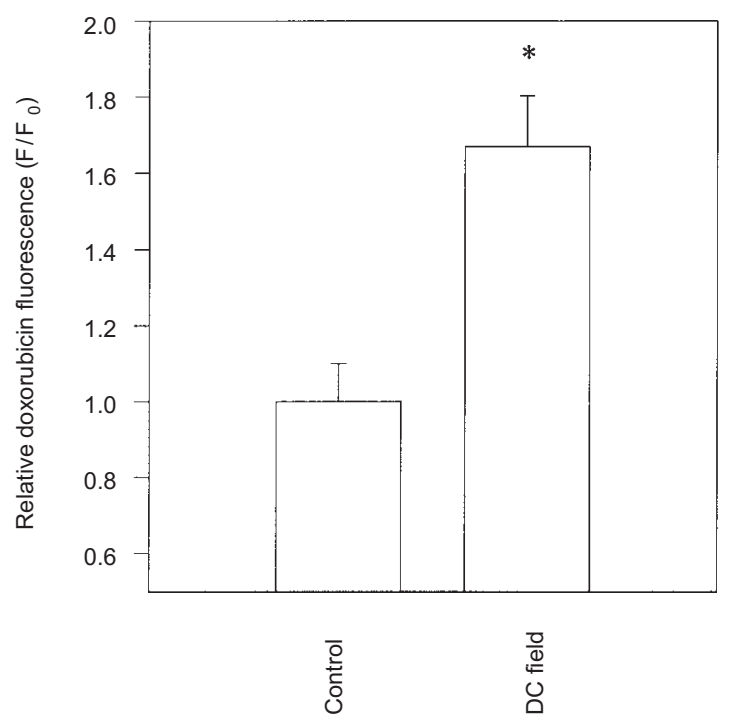

Figure 2 Increased doxorubicin uptake in multicellular tumour spheroids treated with a single electrical field pulse $\left(500 \mathrm{Vm}^{-1}, 90 \mathrm{~s}\right)$ in the presence of $4 \mu \mathrm{M}$ doxorubicin. (A) Doxorubicin distribution in representative spheroids; left side: untreated control spheroid; right side: electrical field treated

spheroid. The images were recorded $90 \mathrm{~min}$ after electrical field application. The bar represents $100 \mu \mathrm{m}$. (B) Relative doxorubicin fluorescence in control and electrical field treated whole-mount multicellular spheroids $90 \mathrm{~min}$ after field application. The doxorubicin fluorescence in the control sample was set to $100 \%$. About 30 spheroids in four independent experiments were used for the determination of each data point. ${ }^{*} P<0.05$, significantly different from control

every $60 \mathrm{~s}$ and were stored automatically to the host memory of the computer unit of the confocal setup. Image analysis was performed as described previously (Wartenberg et al, 1997). For the determination of doxorubicin retention, spheroids treated with electrical fields in the presence of $4 \mu \mathrm{M}$ doxorubicin were transferred to F10 medium supplemented with $4 \mathrm{~mm}$ doxorubicin. Doxorubicin fluorescence was evaluated $90 \mathrm{~min}$ after addition of doxorubicin. Excitation of doxorubicin was performed using the 543-nm line of a helium-neon laser of the confocal setup. Emission was recorded using a longpass LP 570-nm filter set. In the experiments with free radical scavengers, tumour spheroids were preincubated for $2 \mathrm{~h}$ with either $2.5 \mathrm{mM}$ dehydroascorbate (DHA), dissolved in pulsing buffer or for $24 \mathrm{~h}$ with $30 \mu \mathrm{M} \alpha$-tocopherol (vitamin E), dissolved in dimethyl sulphoxide (DMSO). Final DMSO concentrations were $<0.1 \%$.

\section{Determination of spheroid growth}

Multicellular tumour spheroids were treated with a single electrical field pulse $\left(500 \mathrm{Vm}^{-1}, 90 \mathrm{~s}\right)$ either in the presence or absence of $4 \mu \mathrm{M}$ doxorubicin; a third sample was treated with doxorubicin alone; and a negative control remained untreated. After $3 \mathrm{~h}$, the spheroids were washed three times in F10 medium and further incubated for 3 days. Spheroid sizes were determined after washout of doxorubicin prior to the post-incubation period and subsequently after 24 and $72 \mathrm{~h}$. Spheroid volumes were calculated according to $4 / 3 \times \pi \times r_{s}{ }^{2} \times r_{1}$, where $r_{s}$ represents the small radius of the respective spheroids and $r_{1}$ and large radius.

\section{Determination of fluid phase uptake and lethal staining of cells}

For fluid phase uptake studies the hydrophilic, membrane-impermeant tracer Lucifer yellow CH (Sigma, Deisenhofen, Germany) was used. Either multicellular spheroids or single cells enzymatically dissociated from spheroids were suspended in pulsing buffer containing Lucifer yellow in a final concentration of $20 \mu \mathrm{M}$, and transferred to the pulsing chamber mounted to the stage of the confocal setup. Fluid uptake was continuously monitored before, during and after electrical field treatment with field strengths ranging from $500 \mathrm{Vm}^{-1}$ to $3000 \mathrm{Vm}^{-1}$, applied for $90 \mathrm{~s}$. Reversible and irreversible membrane breakdown was evaluated as intracellular increase of Lucifer yellow fluorescence. Excitation was performed using the 488-nm line of the argon-ion laser of the confocal setup. Emission was recorded using a longpass LP515$\mathrm{nm}$ filter set.

Lethal staining was performed using the lethal cell marker Ethidium homodimer-1 (Ethd-1) (Molecular Probes, Eugene, OR, USA). Spheroids were treated with a field strength of $3000 \mathrm{Vm}^{-1}$ for $90 \mathrm{~s}$ and were incubated $15 \mathrm{~min}$ subsequent to electrical field treatment for $30 \mathrm{~min}$ at room temperature with Ethd-1 (final concentration $12 \mu \mathrm{M})$. Excitation of Ethd-1 was performed using the 488-nm line of the argon-ion laser. Emission was recorded using a longpass LP590-nm filter set.

\section{Determination of intracellular redox state}

Intracellular redox state levels were measured using the fluorescent dye $2^{\prime}, 7^{\prime}$-dichlorofluorescein diacetate (H DCFDA) (Molecular Probes), which is a non-polar compound that is converted into a non-fluorescent polar derivative by cellular esterases after incorporation into cells. $\mathrm{H}_{2} \mathrm{DCF}$ is membrane impermeable and is rapidly oxidized to the highly fluorescent $2^{\prime}, 7^{\prime}$-dichlorofluorescein (DCF) in the presence of intracellular ROS (Frenkel and Gleichauf, 1991). For the experiments, multicellular tumour spheroids were incubated in F10 medium containing $20 \mu \mathrm{M} \mathrm{H}_{2}$ DCFDA for $30 \mathrm{~min}$ at room temperature. Spheroids were then washed twice in F10 medium, once in pulsing buffer and transferred to the experimental chamber. For fluorescence excitation, the 488-nm band of the argon ion laser of the confocal setup was used. Emission was recorded using a longpass LP515-nm filter set.

\section{Determination of lipid peroxidation}

Lipid peroxidation of membrane preparations was measured by use of a colorimetrically based kit (Calbiochem-Novabiochem 

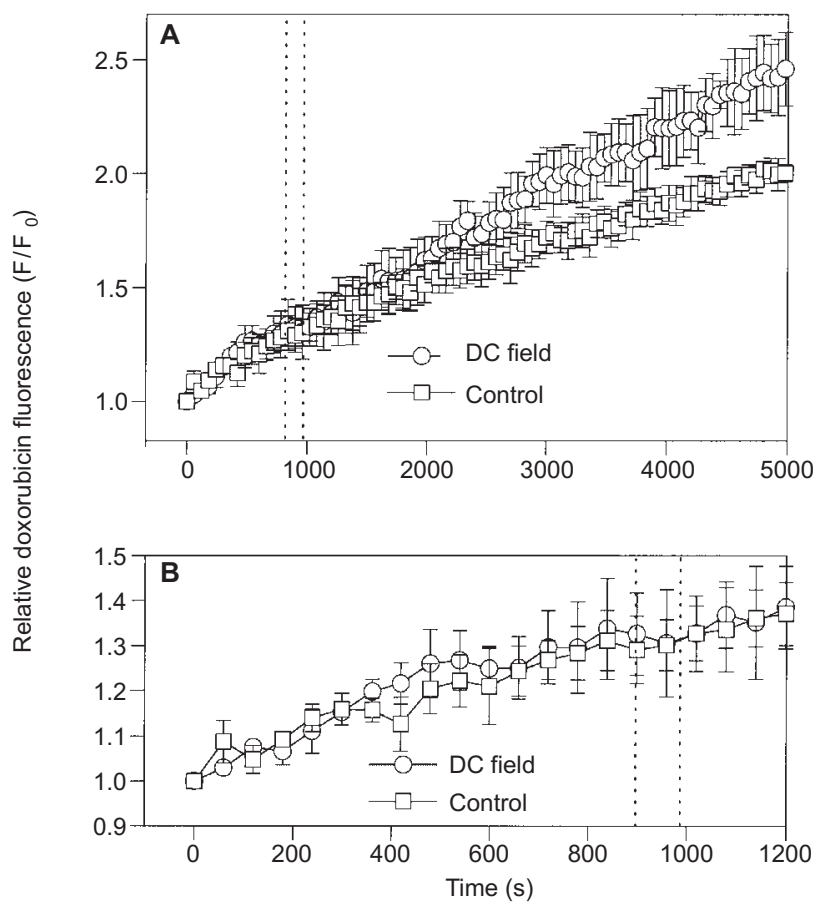

Figure 3 Time course of doxorubicin uptake in multicellular spheroids. (A) Doxorubicin fluorescence increase prior, during and following electrical field $\left(500 \mathrm{Vm}^{-1}, 90 \mathrm{~s}\right)$ treatment. (B) Presentation of doxorubicin fluorescence increase during electrical field treatment. The electrical field was applied during the time indicated by the vertical dashed lines. Note that doxorubicin uptake is not enhanced during electrical field application, indicating that the used field strength did not induce membrane breakdown processes.

International, Inc. San Diego, CA, USA). In brief, membrane preparations of $10^{5}$ cells containing end products of lipid peroxidation, e.g. malonaldehyde and 4-hydroxy-2(E)-nonenal, were reacted with the chromophore $N$-methyl-2-phenylindole and with methanesulphonic acid $\left(40 \mathrm{~min}\right.$ at $\left.45^{\circ} \mathrm{C}\right)$. The samples were cooled on ice and centrifuged at $10000 \mathrm{~g}$ for $5 \mathrm{~min}$ at room temperature. The absorbance of the supernatant at 590-nm and $25^{\circ} \mathrm{C}$ was read on a spectrofluorometer. The combined concentration of malondialdehyde and 4-hydroxy-2(E)-nonenal was determined from a standard curve.

\section{Fluorescence after photobleaching (FRAP) experiments}

FRAP experiments were performed using the line-scanning microphotolysis technique recently developed by Wedekind et al (1996). In brief, electrical field $\left(500 \mathrm{Vm}^{-1}, 90 \mathrm{~s}\right)$-treated and untreated control multicellular tumour spheroids were labelled $2 \mathrm{~h}$ after field exposure for $15 \mathrm{~min}$ with the fluorescent lipid analogue 2-(6-(7-nitrobenz-2-oxa-1,3-diazol-4-yl)amino)hexanoyl-1-hexadecanoyl-sn-glycero-3-phosphocholine (NBD-C 6 -HPC) (Molecular Probes) dissolved in a final concentration of $10 \mu \mathrm{g} \mathrm{ml}^{-1}$ in E1 buffer containing $135 \mathrm{~mm}$ sodium chloride, $5.4 \mathrm{~mm}$ potassium chloride, $1.8 \mathrm{mM}$ calcium chloride, $1 \mathrm{~mm}$ magnesium chloride, $10 \mathrm{~mm}$ glucose, $10 \mathrm{~mm}$ HEPES ( $\mathrm{pH} 7.4$ at $23^{\circ} \mathrm{C}$ ). They were subsequently washed three times in E1 buffer and transferred to an incubation chamber mounted on the stage of the confocal setup. The initial fluorescence before photobleaching was evaluated by recording a $512 \times 512$ pixel $x, y$ scan image. From this prebleach image the mean

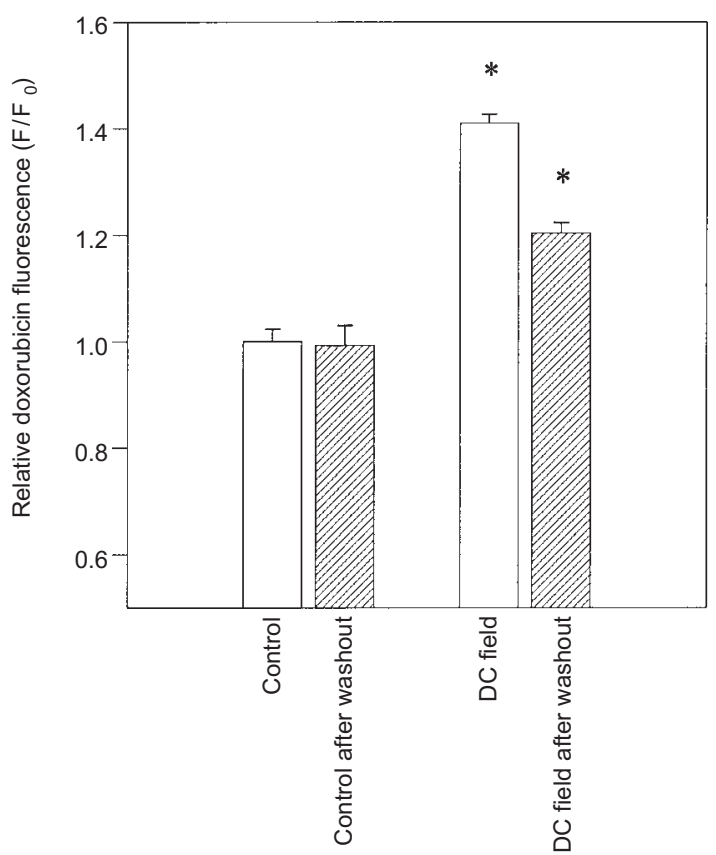

Figure 4 Doxorubicin retention in electrical field treated $\left(500 \mathrm{Vm}^{-1}, 90 \mathrm{~s}\right)$ multicellular spheroids. Electrical field treated spheroids (hatched bars) and an untreated control (open bars) were incubated with $4 \mu \mathrm{m}$ doxorubicin during electrical field application and post-incubated in the presence of doxorubicin for $90 \mathrm{~min}$. Thereafter, external doxorubicin was washed out and doxorubicin fluorescence was evaluated following an incubation period of $90 \mathrm{~min}$ in doxorubicin-free F10 medium. About 30 spheroids in three independent experiments were used for the determination of each data point. ${ }^{*} P<0.05$, significantly different from control

fluorescence was determined in the region of interest predisposed for photobleaching. The confocal microscope was subsequently switched to the line scan mode and the laser power was increased from $2.5 \mathrm{~mW}$ to $25 \mathrm{~mW}$. During line scanning, which was performed for $3 \mathrm{~s}$, the laser beam was repetitively scanned at high speed ( $3 \mathrm{~ms}$ per line scan) along a selected line. After photolysis along the selected line, full frame images $(512 \times 512$ pixel $)$ were recorded in intervals of $15 \mathrm{~s}$, and the fluorescence recovery in the centre of the line was determined using an overlay mask. The data were plotted for display by non-linear least-squares fit of the data and the lipid lateral diffusion coefficient was calculated from the recovery curves according to Koppel et al (1979) who described the relationship between fluorescence $\mathrm{F}$ at the scanning point $\Delta \mathrm{x}$ from the centre of the bleached area at time $t$ by the following equations

$\mathrm{F}(\Delta \mathrm{x}, t)=\mathrm{F}_{0}\left\{\left[1-\beta \alpha_{2}(t)\right] \exp \left[-(\Delta \mathrm{x})^{2} / \omega^{2}(t)\right]\right\}-\left\{(1-\beta) \alpha_{0} \exp \left[-(\Delta \mathrm{x})^{2} / \omega_{0}^{2}\right]\right\}(1)$ $\alpha_{2}=\alpha_{0} /\left(1+\mathrm{t} / \tau_{\mathrm{D}}\right)$

$\tau_{\mathrm{D}}=\omega_{0}^{2 / 4 \mathrm{D}}$

where $\beta$ represents the size of the mobile fraction, $\alpha_{0}$ is a constant that characterizes the extent of bleaching, $\omega_{0}$ is the $1 / e$ radius of the laser beam size, $\tau_{\mathrm{D}}$ is the diffusion time, and $D$ is the diffusion coefficient.

\section{Statistical analysis}

Data are given as mean values \pm s.e.m. with $n$ denoting the number of experiments. Student's $t$-test for unpaired data was applied as appropriate. A value of $P<0.05$ was considered significant. 


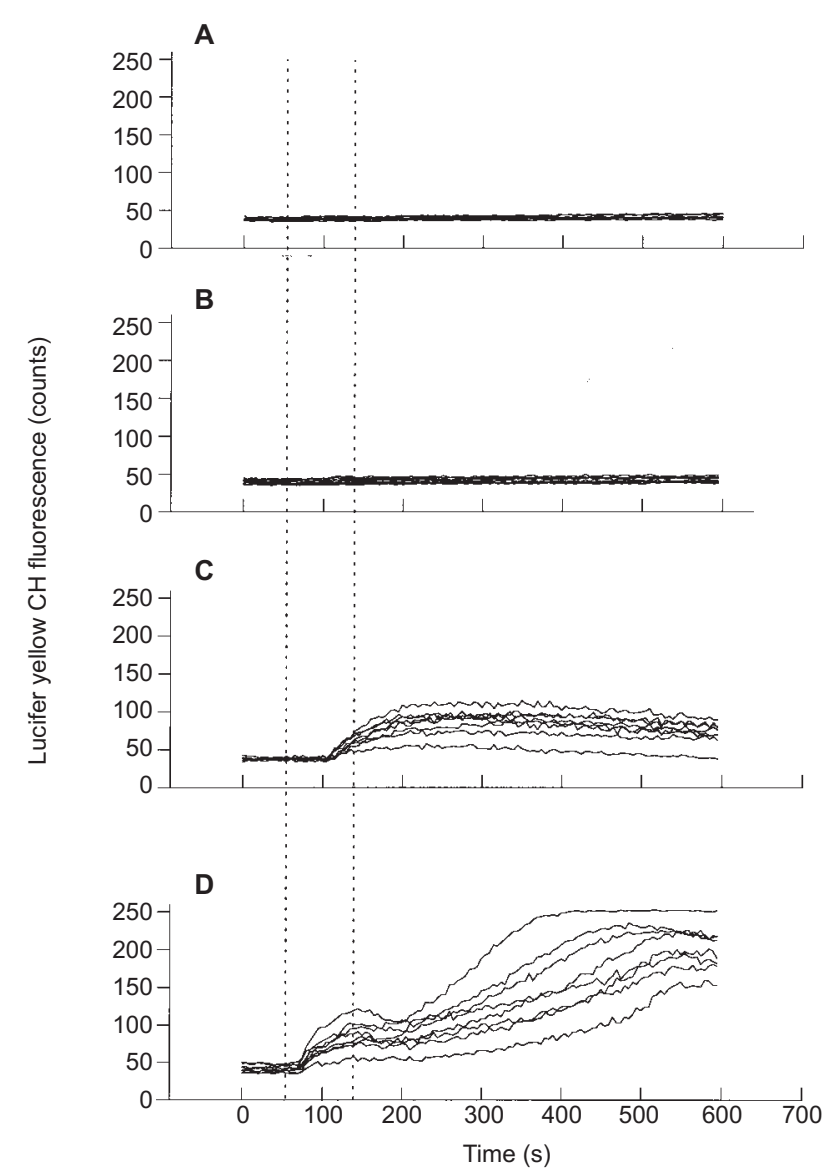

Figure 5 Time course of fluid phase uptake of Lucifer yellow $\mathrm{CH}$ in single cells treated with different field strengths. Single cells were enzymatically dissociated from multicellular tumour spheroids. (A) Control; (B) $500 \mathrm{Vm}^{-1}$, $90 \mathrm{~s}$; (C) $1500 \mathrm{Vm}^{-1}, 90 \mathrm{~s}$; (D) $2000 \mathrm{Vm}^{-1}, 90 \mathrm{~s}$. The electrical field was applied during the time indicated by the vertical dashed lines

\section{RESULTS}

\section{Enhanced doxorubicin uptake and retention in multicellular tumour spheroids treated with electrical fields}

When multicellular tumour spheroids were treated with a single electrical field pulse with a field strength of $500 \mathrm{Vm}^{-1}$ and a duration of $90 \mathrm{~s}$, which has previously been shown to stimulate tumour growth (Sauer et al, 1997; Wartenberg et al, 1997), a significant increase by $67 \pm 3 \%$ of doxorubicin uptake (Figure 2 A,B) was observed after $90 \min (n=4)$. Doxorubicin fluorescence was homogenously distributed within the tissue, i.e. no preferential accumulation at the cathode- or anode-facing was observed. The time course of doxorubicin uptake was monitored by CLSM during electrical field treatment. Figure 3 A,B shows that doxorubicin uptake is not enhanced during, but following, the electrical field pulse, indicating that the observed effect is not owing to either reversible or irreversible membrane breakdown $(n=3)$. The observed increase in doxorubicin uptake after electrical field treatment may be at least partially owing to reduced doxorubicin efflux. Therefore, doxorubicin retention was assessed in electrical field-treated tumour spheroids $90 \mathrm{~min}$ after doxorubicin removal from the external medium. Figure 4 shows that in the electrical field-treated sample a significant efflux of doxorubicin after washout of external doxorubicin occurred. This

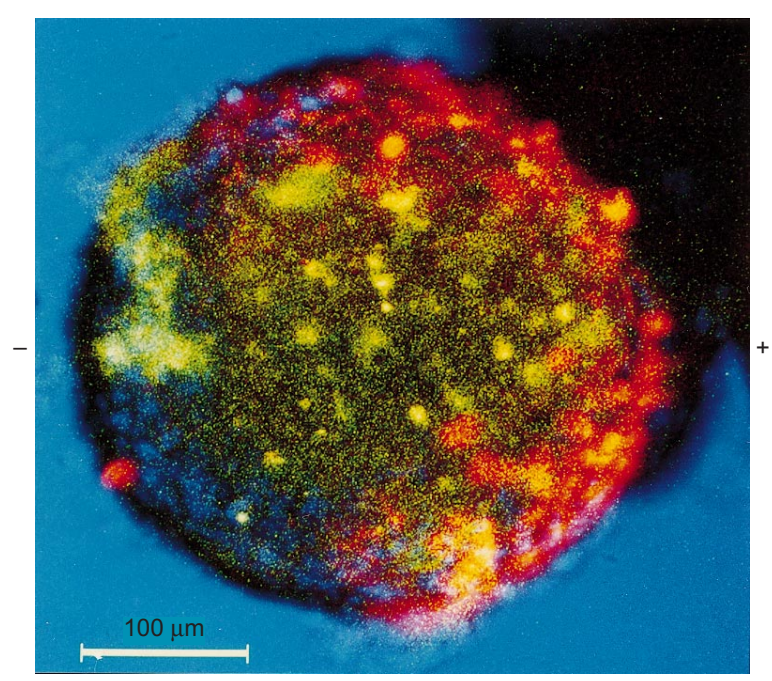

Figure 6 Lucifer yellow $\mathrm{CH}$ fluid phase uptake and Ethd-1 lethal staining in a representative multicellular tumour spheroid treated with a $3000 \mathrm{Vm}^{-1}, 90 \mathrm{~s}$ electrical field. Shown is a false colour overlay of a transmission image (blue), a Lucifer yellow fluorescence image (green), and a Ethd-1 fluorescence image (red). Note that cell lethality is increased at the anodefacing side of the spheroid. The bar represents $100 \mu \mathrm{m}$

indicates that the observed enhancement of doxorubicin retention following electrical field treatment may be owing to physical changes in membrane permeability which affect both efflux and influx kinetics of doxorubicin. However, doxorubicin retention after washout of external doxorubicin was still about $20 \%$ enlarged in the electrical field treated sample as compared to control $(n=3)$ which presumably results from intracellular binding of the compound.

To exclude that either reversible or irreversible membrane breakdown occurred during and after electrical field treatment, spheroids and single cells enzymatically dissociated from spheroids were treated with different field strengths in the presence of the polar fluorescence dye Lucifer yellow $\mathrm{CH}$. Membrane breakdown processes should result in an increased uptake of this fluid phase marker. Figure 5 demonstrates that no fluid phase uptake of Lucifer yellow $\mathrm{CH}$ occurred when field strengths of $500 \mathrm{Vm}^{-1}$ and $1000 \mathrm{Vm}^{-1}$ (not shown) were applied for $90 \mathrm{~s}$. A marked fluid phase uptake owing to electrical field treatment was observed with a field strength of $1500 \mathrm{Vm}^{-1}$. The Lucifer yellow uptake occurred during electrical field application and remained on a sustained plateau, indicating reversible membrane breakdown $(n=3)$. Treatment with a field pulse of $2000 \mathrm{Vm}^{-1}$ resulted in fluid phase uptake during field application, followed by a further rise of Lucifer yellow fluorescence shortly after treatment, which is indicative for irreversible membrane breakdown $(n=3)$. When multicellular tumour spheroids were treated with an electrical field pulse of $3000 \mathrm{Vm}^{-1}$ and Lucifer yellow staining was co-localized with Ethd-1 staining of lethal cells, an increased cell lethality was observed at the anode-facing pole of the spheroid, indicating more pronounced cell membrane potential changes resulting in irreversible membrane breakdown processes (Figure 6) $(n=3)$.

\section{Doxorubicin toxicity is increased after DC electrical field treatment of multicellular tumour spheroids}

To test whether adjuvant DC electrical field treatment $\left(500 \mathrm{Vm}^{-1}\right.$, $90 \mathrm{~s}$ ) augmented doxorubicin toxicity, multicellular tumour spheroids were electrical field-treated in the presence of doxorubicin, and 


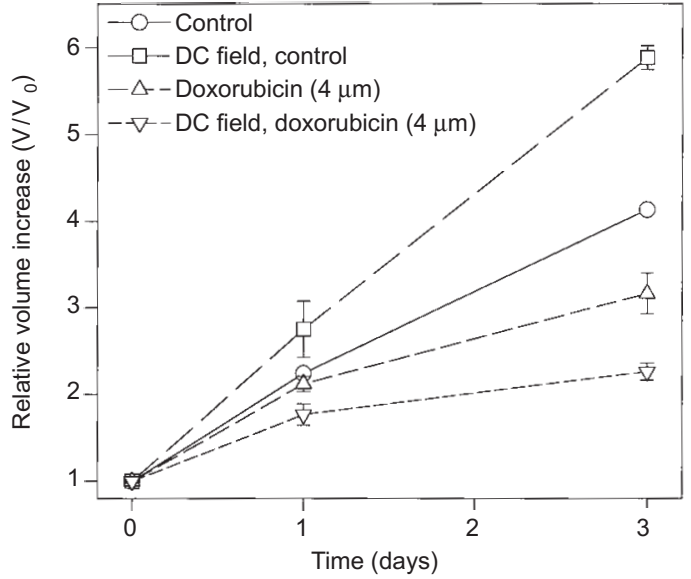

Figure 7 Growth kinetics of multicellular tumour spheroids after electrical field $\left(500 \mathrm{Vm}^{-1}, 90 \mathrm{~s}\right)$ treatment. One sample of spheroids was treated with a single electrical field pulse $\left(500 \mathrm{Vm}^{-1}, 90 \mathrm{~s}\right)$ in the presence of doxorubicin $(4 \mu \mathrm{M})(\nabla)$. A second sample was electrical field treated in the absence of doxorubicin $(\square)$. A third sample was treated with doxorubicin $(4 \mu \mathrm{M})$ alone $(\triangle)$. A control sample remained untreated $(\bigcirc)$. Note that electrical field treatment in the absence of doxorubicin enhanced the growth kinetics of tumour spheroids as previously reported (Sauer et al, 1997)

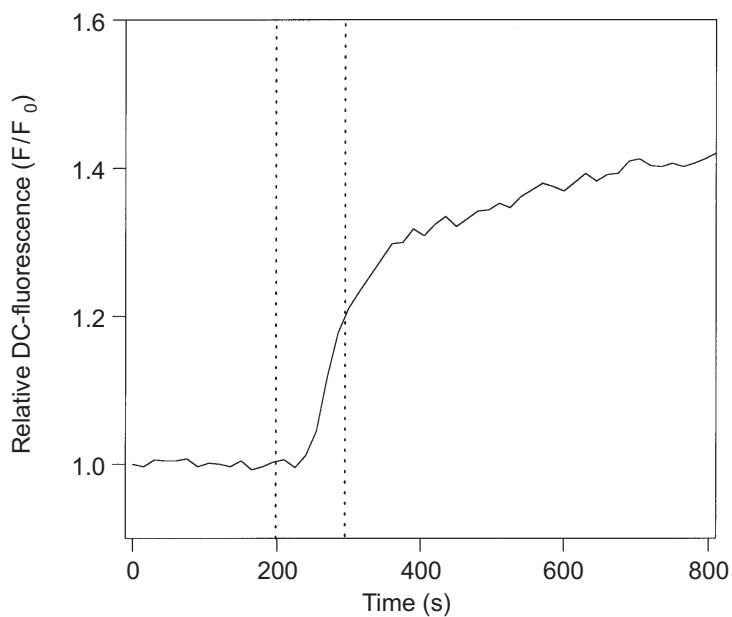

Figure 8 ROS generation during and following treatment of multicellular tumour spheroids with a single electrical field pulse $\left(500 \mathrm{Vm}^{-1}, 90 \mathrm{~s}\right)$.

Representative trace of normalized dichlorofluorescein (DCF) fluorescence. The electrical field was applied during the time indicated by the dashed lines

spheroid growth was monitored. Figure 7 shows the growth kinetics of control spheroids, spheroids treated with doxorubicin alone and spheroids treated with a single electrical field pulse $\left(500 \mathrm{Vm}^{-1}, 90 \mathrm{~s}\right)$ in the presence or absence of doxorubicin. As reported previously (Sauer et al, 1997; Wartenberg et al, 1997), the electrical field pulse led to an enhancement of tumour growth. However, electrical field treatment in the presence of doxorubicin resulted in a significantly decreased growth kinetics as compared to spheroids treated with doxorubicin alone $(n=3)$.

\section{The increased doxorubicin uptake is owing to the generation of intracellular reactive oxygen species by electrical field treatment}

To investigate underlying mechanisms for the observed increase of doxorubicin uptake following electrical field treatment, intracellular

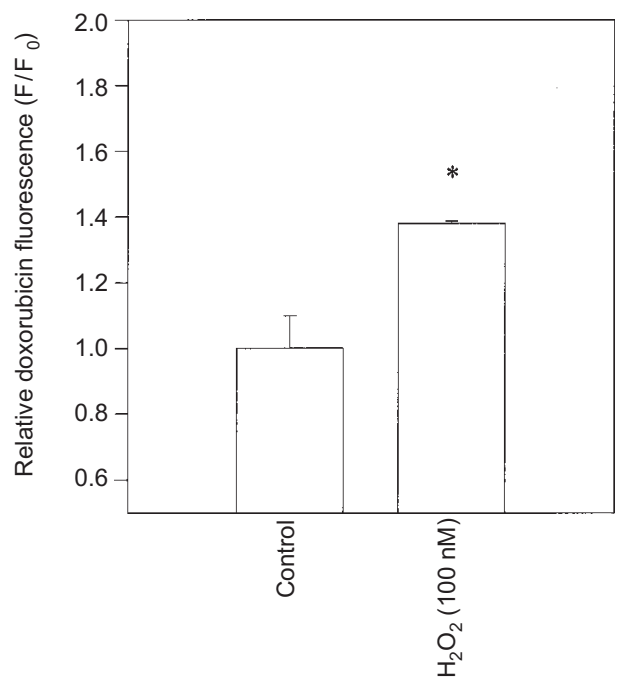

Figure 9 Effect of 100 nm hydrogen peroxide on the doxorubicin uptake in multicellular spheroids. Spheroids were treated for 90 min with $\mathrm{H}_{2} \mathrm{O}_{2}$ in the presence of doxorubicin and doxorubicin fluorescence was evaluated in whole-mount spheroids. About 30 spheroids in four independent experiments were used for the determination of each data point. ${ }^{*} P<0.05$, significantly different from control

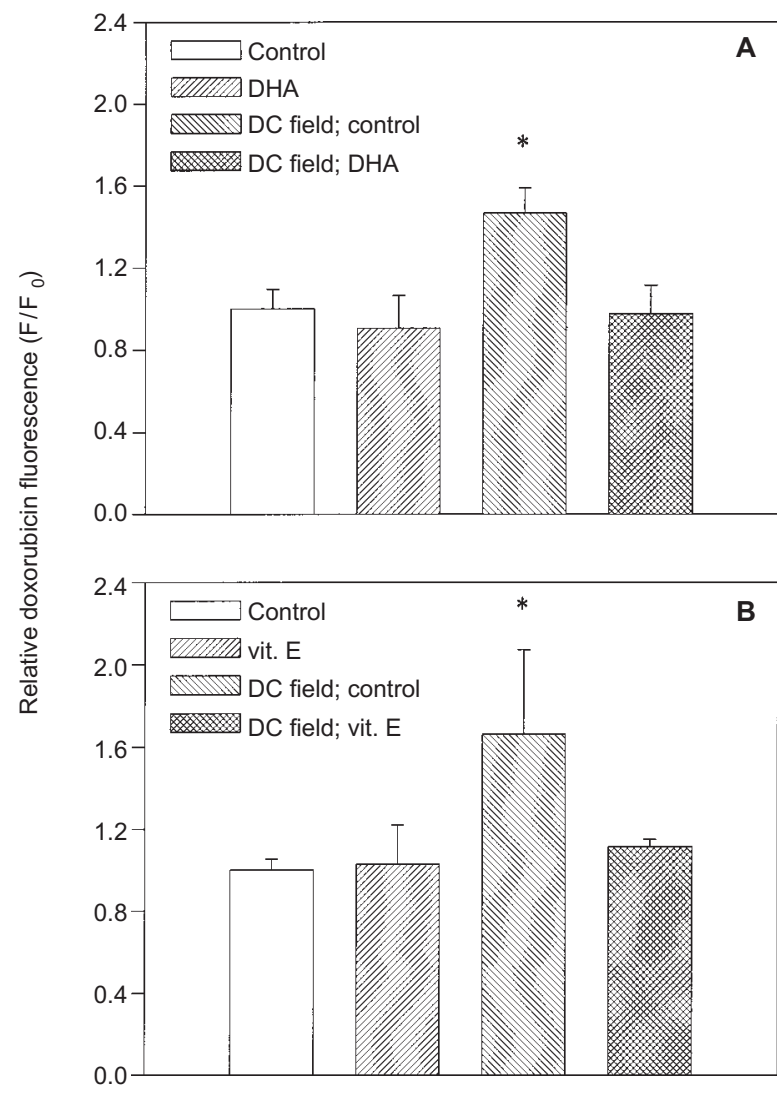

Figure 10 Effects of the radical scavengers DHA $(2.5 \mathrm{~mm})(\mathbf{A})$ and $\alpha$ tocopherol (vitamin E) $(30 \mu \mathrm{M})(B)$ on doxorubicin uptake following electrical field $\left(500 \mathrm{Vm}^{-1}, 90 \mathrm{~s}\right)$ treatment. Doxorubicin fluorescence was determined after an incubation period of $90 \mathrm{~min}$ in: (1) an untreated control; (2) an untreated control preincubated for $2 \mathrm{~h}$ and $24 \mathrm{~h}$ with DHA and $\alpha$-tocopherol respectively; (3) an electrical field treated sample; (4) in an electrical field treated sample after preincubation with radical scavengers. About 30 spheroids in three independent experiments were used for the determination of each data point. ${ }^{*} P<0.05$, significantly different from control 


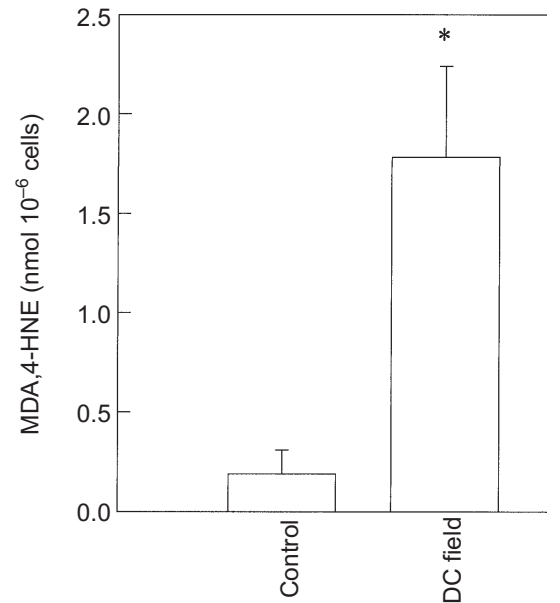

Figure 11 Determination of the lipid peroxidation end-products 4-HNE and MDA in cell extracts from multicellular tumour spheroids treated with a single electrical field pulse $\left(500 \mathrm{Vm}^{-1}, 90 \mathrm{~s}\right)$. Evaluation of lipid peroxidation was performed $2 \mathrm{~h}$ after electrical field treatment. ${ }^{\star} P<0.05$, significantly different from control reactive oxygen species (ROS) were determined using DCF as an indicator. Figure 8 shows that during electrical field treatment ROS were generated $(n=5)$. The amount of ROS generated was equivalent to the DCF signal elicited with 50-100 nM hydrogen peroxide (Wartenberg et al, 1997). Consequently, nanomolar concentrations (100 nM) of externally added hydrogen peroxide mimicked the effect of the electrical field on doxorubicin uptake (see Figure 9), indicating that ROS generation subsequent to electrical field treatment is sufficient to enhance doxorubicin uptake $(n=4)$. This was further corroborated by experiments where multicellular tumour spheroids were preincubated with the radical scavengers dehydroascorbate (DHA) $(2.5 \mathrm{~mm})$ and $\alpha$-tocopherol (vitamin E) $(30 \mu \mathrm{M})$. Figure 10 A,B clearly demonstrates that both DHA and vitamin E (dissolved in DMSO) present during electrical field treatment reduced doxorubicin uptake to the control level of the untreated sample $(n=5)$, indicating that ROS generation following electrical field treatment of multicellular tumour spheroids is mediating the enhancement of doxorubicin uptake. DMSO in the absence of $\alpha$-tocopherol did not exert any effect on the electrical field-induced doxorubicin accumulation (data not shown).
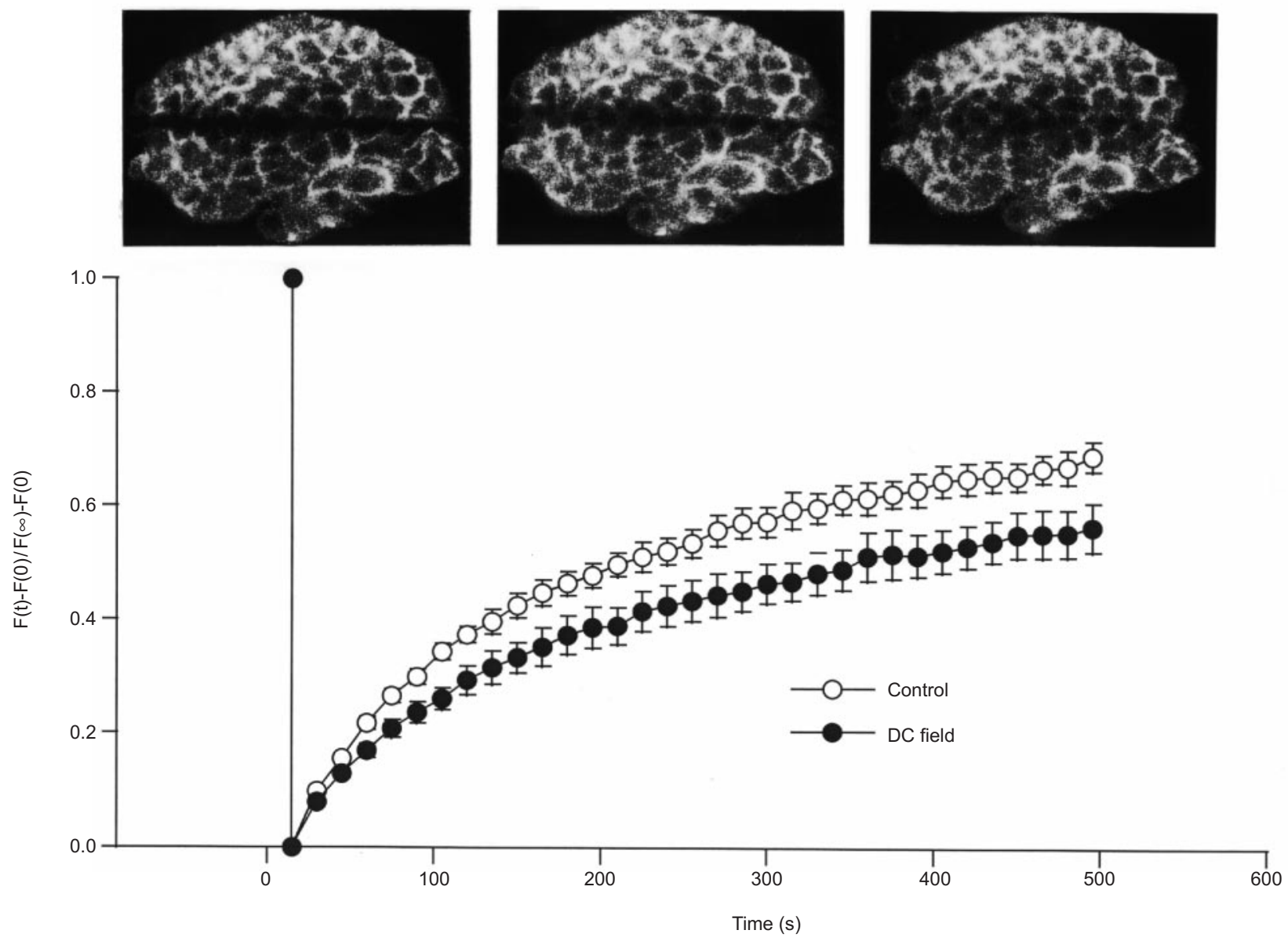

Figure 12 Lipid lateral diffusion is decreased in multicellular tumour spheroids treated with a single electrical field pulse (500 Vm ${ }^{-1}$, $\left.90 \mathrm{~s}\right)$. FRAP experiments were performed with spheroids stained with the fluorescent lipid analogue NBD-C -HPC. Photobleaching along a preselected line was achieved by using the line-scanning mode of the confocal microscope. The fluorescence images show (from the left to the right) the fluorescence recovery along the photobleached line in a representative tumour spheroid $0 \mathrm{~s}, 150 \mathrm{~s}$ and $300 \mathrm{~s}$ after photobleaching. The traces show one of four experiments with comparable results. The fluorescence recovery of at least three spheroids was determined to give one data point 


\section{ROS generated during electrical field treatment of multicellular tumour spheroids induce membrane lipid peroxidation}

ROS generated during electrical field treatment of multicellular tumour spheroids may induce membrane lipid peroxidation, resulting in an alteration of the permeability properties of the cell membrane for doxorubicin. To assess lipid peroxidation malondialdehyde (MDA) and 4-hydroxy-2(E)-nonenal (4-HNE), which are end products of lipid peroxidation, were evaluated in cell extracts of electrical field treated multicellular spheroids $2 \mathrm{~h}$ after electrical field treatment $(n=3)$. Figure 11 shows that MDA and 4HNE in a concentration of $1.78 \pm 0.26 \mathrm{nmol} 10^{-6}$ cells were generated following electrical field treatment, indicating membrane lipid peroxidation, which presumably resulted in changes of lipid bilayer structure mediating increased doxorubicin uptake.

\section{Electrical field-induced membrane rigidization}

Lipid peroxidation following electrical field treatment of multicellular tumour spheroids may alter the fluidity of cell membranes, which may have effects on doxorubicin uptake and efflux kinetics. To evaluate electrical field-induced changes in lipid mobility FRAP experiments were performed using the fluorescent lipid analogue NBD- $\mathrm{C}_{6}-\mathrm{HPC}$ and the line-scanning mode of the CLSM. Our data show that fluorescence recovery after photobleaching was reduced (Figure 12) when multicellular tumour spheroids had been treated with an electrical field pulse $2 \mathrm{~h}$ prior to the FRAP experiments, which resulted in a significantly decreased diffusion coefficient D with $4.2 \times 10^{-10} \pm 1 \times 10^{-10} \mathrm{~cm}^{2} \mathrm{~s}^{-1}$ as compared to $2.7 \times 10^{-10} \pm 0.6 \times 10^{-10} \mathrm{~cm}^{2} \mathrm{~s}^{-1}$ in the untreated control group ( $n=13$ spheroids from four different spheroid cultures).

\section{Discussion}

The electrical field strengths used in current approaches of ECT are markedly above the critical field strengths for reversible or irreversible membrane breakdown (Domenge et al, 1996; Heller et al, 1997). Therefore, the enhancement of drug delivery following electrical field treatment is thought to be mediated by electroporation processes (Belehradek et al, 1994). Owing to severe sideeffects of the electrical fields on non-cancerous cells, the applicability of ECT is reduced to the close vicinity of tumours.

The non-lethal low field strength $\left(500 \mathrm{Vm}^{-1}, 90 \mathrm{~s}\right)$ applied in the present study has previously been used by us to enhance the growth kinetics of multicellular tumour spheroids and to recruit quiescent cells in the depth of the tumour tissue for the cell cycle (Sauer et al, 1997; Wartenberg et al, 1997). We have chosen this proliferation-inducing electrical field strength since proliferationactive cells have been demonstrated to be more susceptible towards treatment with anticancer agents than dormant cells, which have been reported to display features of resistance both towards radiation and chemotherapy (Remvikos et al, 1993; Olive and Durand, 1994; Hietanen et al, 1995; Wartenberg et al, 1998). An approach using low field-strength non-toxic electrical fields for ECT should be promising and may result in an increased efficacy of chemotherapy. However, chemosensitization by stimulation of tumour cell proliferation should assure the best possible access of administered cytostatics to the tumours in order to avoid a fatal ECT-induced enhancement in tumour growth.

The data of the present study demonstrate that treatment of multicellular tumour spheroids with a single electrical field pulse of low field-strength $\left(500 \mathrm{Vm}^{-1}, 90 \mathrm{~s}\right)$ significantly increased doxorubicin uptake and, consequently, doxorubicin toxicity. We could show that the applied electrical field strength did not lead to either reversible or irreversible membrane breakdown processes since - as described previously (Sauer et al, 1997) - a field strength of $500 \mathrm{Vm}^{-1}$ applied to a spherical object of approximately $150 \mu \mathrm{m}$ in diameter resulted in a membrane potential change of $75 \mathrm{mV}$, which is far below the critical membrane potential change of $0.5-1.5 \mathrm{~V}$ that has been reported to be essential to induce membrane breakdown processes (Weaver, 1993). Moreover, fluid phase uptake studies with the hydrophilic fluorescence probe Lucifer yellow $\mathrm{CH}$ showed that fluid phase uptake was not observed in single cells when field strengths of $500 \mathrm{Vm}^{-1}(90 \mathrm{~s})$ were applied. Experiments with different field strengths revealed that reversible membrane breakdown occurred with a critical electrical field strength of $1500 \mathrm{Vm}^{-1}$ (90 s), which resulted in a sustained rise in Lucifer yellow uptake during and shortly following electrical field. Irreversible membrane breakdown was observed at a field strength of $2000 \mathrm{Vm}^{-1}$ (90 s) and was characterized by an initial rise of Lucifer yellow fluorescence during electrical field treatment, which was followed by a longlasting fluid phase uptake. As previously described (Sauer et al, 1997), lethal effects of the electrical field were found with a critical field strength of $3000 \mathrm{Vm}^{-1}$. Interestingly, cell lethality as indicated by Ethd-1 lethal cell staining was mainly observed at the anode-facing pole of the spheroid. This effect can be explained by a superimposition of the electrical field vector of the cell membrane potential and the electrical field vector of the external electrical field, which are acting in the same direction and lead to enlarged membrane potential changes at the anode-facing as compared to the cathode-facing side of the spheroid (Sauer et al, 1997).

Electrical fields have been shown to change the intracellular redox state (Wartenberg et al, 1997). An increase in intracellular ROS may lead to lipid peroxidation and to altered permeability properties of the cell membrane (Stanimirovic et al, 1995). Changes in the intracellular redox state occurred during electrical field treatment with a $500 \mathrm{Vm}^{-1}(90 \mathrm{~s})$ electrical field using the fluorescence indicator DCF. Furthermore, the lipid peroxidation products MDA and 4-HNE were detected in the cell homogenates of tumour spheroids that had been treated with $500 \mathrm{Vm}^{-1}(90 \mathrm{~s})$ electrical fields. To investigate whether electrical field generated ROS were a prerequisite for the enhancement of doxorubicin uptake, spheroids were incubated in the absence of an external electrical field with $100 \mathrm{~nm}$ hydrogen peroxide, which resulted in a doxorubicin uptake not significantly different from the uptake observed after electrical field treatment, indicating that the electrical field-generated ROS were equivalent to $100 \mathrm{nM}$ externally added hydrogen peroxide, as previously reported (Wartenberg et al, 1997). Moreover, incubation of multicellular tumour spheroids with DHA, a radical scavenger of cytoplasmic ROS, and $\alpha$-tocopherol, which inhibits lipid peroxidation by integration into the plasma membrane (Susa et al, 1996), abolished the electrical fieldinduced enhancement of doxorubicin uptake, indicating that the observed effect was owing to changes in the cellular oxidized state.

The data of the present study suggest that lipid peroxidation following electrical field treatment results in an increased membrane permeability for doxorubicin, which affects both the uptake and the efflux of the drug, but as a net effect results in increased doxorubicin retention, presumably owing to intracellular binding. It has been previously shown that lipid peroxidation results in membrane rigidization with the consequence of altered permeation properties of the cell membrane (Deliconstantinos et 
al, 1996; Garcia et al, 1997; Verstraeten et al, 1997). These data were corroborated by the FRAP experiments of the present study, which show that electrical field treatment of tumour spheroids significantly decreased membrane fluidity, presumably owing to the ROS generated by electrical field treatment.

The parameters that govern doxorubicin uptake in cells are still a matter of debate. It has been shown that doxorubicin binds to anionic phospholipids in the membrane which involves electrostatic interaction as well as penetration of the electrostatically bound drug between the acyl chains (de Wolf et al, 1993; Speelmans et al, 1994). The uncharged form of the drug is transported via passive diffusion, of which the driving force is the intracellular acid $\mathrm{pH}$ and drug binding to intracellular targets (Harrigan et al, 1993). In the cytoplasmic compartment, doxorubicin has been demonstrated to generate ROS by doxorubicin quinone reduction and redox-cycling (Keizer et al, 1990).

Significant scientific efforts have been undertaken to investigate the cytotoxic effects of ROS generated by doxorubicin redoxcycling, which have been related to ROS-dependent DNA damage and lipid peroxidation with the consequence of impaired signal transduction pathways (Keizer et al, 1990; Cummings et al, 1992). However, the influence of the intracellular oxidized state and lipid peroxidation on doxorubicin uptake is not well-documented. In a recent study, doxorubicin toxicity was found to be enhanced after incubation of breast cancer cells with polyunsaturated fatty acids, which are known to induce lipid peroxidation. Incubation with radical scavengers caused cell viability to return to the baseline level of doxorubicin in the absence of polyunsaturated fatty acids (Germain et al, 1998). Moreover, in a doxorubicin-resistant mammary tumour cell line, lipid peroxidation levels were found to be lower than in the wild-type cell line (Benchekroun et al, 1993). These data point towards the notion that lipid peroxidation may be a general mechanism by which the permeability of tumour cell membranes may be increased for doxorubicin and its anthracycline derivatives.

The ECT approach elaborated in the present study for the in vitro model of multicellular tumour spheroids demonstrates that electrical field strengths far below the threshold of membrane breakdown processes effectively increase doxorubicin uptake in the tumour tissue. Changing the intracellular oxidized state of tumours, or tumour-bearing organs, by low field-strength electrical fields may be useful to chemosensitize tumours for chemotherapeutic treatment. Since electrical fields can be applied locally, an increased doxorubicin toxicity in the target area may be achieved with lower doses of orally or intravenously administered drug as are currently applied during conventional chemotherapy.

The in vitro approach of the present study may initiate in vivo investigations with the aim of selectively increasing anthracycline uptake in the diseased organ rather than the whole body, which when lower doses of the cytostatic are applied - should result in less severe side-effects for the patient.

\section{ACKNOWLEDGEMENTS}

This work is part of the MD thesis of Volker Pütz. We gratefully acknowledge support by the Köln Fortune Programm, University of Cologne, Germany.

\section{REFERENCES}

Belehradek M, Domenge C, Luboinski B, Orowski S, Belehradek J Jr and Mir LM (1993) Electrochemotherapy, a new antitumor treatment. First clinical phase I-II trial. Cancer 72: 3694-3700
Belehradek J Jr, Orlowski S, Ramirez LH, Pron G, Poddevin B and Mir LM (1994) Electropermeabilization of cells in tissues assessed by qualitative electroloading of bleomycin. Biochim Biophys Acta 1190: 155-163

Benchekroun NM, Pourquier P, Schott B and Robert J (1993) Doxorubicin-induced lipid peroxidation and glutathione peroxidase activity in tumor cell lines selected for resistance to doxorubicin. Eur J Biochem 211: 141-146

Cameron IL, Hardman WE, Winters WD, Zimmerman S and Zimmerman AM (1993) Environmental magnetic fields: influences on early embryogenesis J Cell Biochem 51: 417-425

Cummings J, Willmott N, Hoey BM, Marley ES and Smyth JF (1992) The consequences of doxorubicin quinone reduction in vivo in tumour tissue. Biochem Pharmacol 44: 2165-2174

de Wolf FA, Staffhorst RW, Smits HP, Onwezen MF and Kruijff B (1993) Role of anionic phospholipids in the interaction of doxorubicin and plasma membrane vesicles: drug binding and structural consequences in bacterial systems. Biochemistry 32: 6688-6695

Deliconstantinos G, Villiotou V and Stavrides JC (1996) Tumour promotor tertbutyl-hydroperoxide induces peroxynitrite formation in human erythrocytes. Anticancer Res 16: 2969-2979

Domenge C, Orlowski S, Luboinski B, De Baere T, Schwaab G, Belehradek J Jr and Mir LM (1996) Antitumor electrochemotherapy: new advances in the clinical protocol. Cancer 77: 956-963

Frenkel K and Gleichauf C (1991) Hydrogen peroxide formation by cells treated with a tumour promotor. Free Rad Res Comms 12-13: 783-794

Garcia JJ, Reiter RJ, Guerrero JM, Escames G, Yu BP, Oh CS and Munoz-Hoyos A (1997) Melatonin prevents changes in microsomal membrane fluidity during induced lipid peroxidation. FEBS Lett 26: 297-300

Germain E, Chajes V, Cognault S, Lhuillery C and Bougnoux P (1998) Enhancement of doxorubicin toxicity by polyunsaturated fatty acids in the human breast tumor cell line MDA-MB-231: relationship to lipid peroxidation. Int J Cancer 75: $578-583$

Glass LF, Pepine ML, Fenske NA, Jaroszeski M, Reintgen DS and Heller R (1996) Bleomycin-mediated electrochemotherapy of metastatic melanoma. Arch Dermatol 132: 1353-1357

Harrigan PR, Wong KF, Redelmeier TE, Wheeler JJ and Cullis PR (1993) Accumulation of doxorubicin and other lipophilic amines into large unilamellar vesicles in response to transmembrane $\mathrm{pH}$ gradients. Biochim Biophys Acta 1149: $329-338$

Heller R, Jaroszeski MJ, Glass LF, Messina JL, Rapaport DP, DeConti RC, Fenske NA, Gilbert RA, Mir LM and Reintgen DS (1996) Phase I/II trial for the treatment of cutaneous and subcutaneous tumours using electrochemotherapy. Cancer 77: 964-971

Heller R, Jaroszeski M, Perrot R, Messina J and Gilbert R (1997) Effective treatment of B16 melanoma by direct delivery of bleomycin using electrochemotherapy. Melanoma Res 7: 10-18

Hietanen P, Blomqvist C, Wasenius VM, Niskanen E, Frarnssila K and Nordling S (1995) Do DNA ploidy and S-phase fraction in primary tumour predict the response to chemotherapy in metastatic breast cancer? Br J Cancer 71: 1029-1032

Keizer HG, Pinedo HM, Schuurhuist GJ and Joenje H (1990) Doxorubicin (adriamycin): a critical review of free radical-dependent mechanisms of cytotoxicity. Pharmac Ther 47: 219-231

Koppel DE (1979) Fluorescence redistribution after photobleaching. Biophys $J$ 28: 281-292

Mir LM, Devauchelle P, Quintin-Colonna F, Delisle F, Dolinger S, Fradelizi D, Belehradek J Jr and Orlowski S (1997) First clinical trial of cat soft-tissue sarcomas treatment by electrochemotherapy. Br J Cancer 76: 1617-1622

Nishi T, Dev SB, Yoshizato K, Kuratsu J and Ushio Y (1997) Treatment of cancer using pulsed electric field in combination with chemotherapeutic agents or genes. Human Cell 10: 81-86

Olive PL and Durand RE (1994) Drug and radiation resistance in spheroids: cell contact and kinetics. Cancer Metastas Rev 13: 121-138

Poddevin B, Orlowski S, Belehradek J Jr and Mir LM (1991) Very high cytotoxicity of bleomycin introduced into the cytosol of cells in culture. Biochem Pharmacol 42: S67-S75

Ramirez LH, Orlowski S, An D, Bindloula G, Dzodic R, Ardouin P, Bognel C, Belehradek J Jr, Munck J-N and Mir LM (1998) Electrochemotherapy on liver tumours in rabbits. Br J Cancer 77: 2104-2111

Remvikos Y, Mosseri V, Zajdela A, Fourquet A, Durand JC, Pouillard P and Magdelenat H (1993) Prognostic value of the S-phase fraction of breast cancers treated by primary radiotherapy or neoadjuvant chemotherapy. Ann NY Acad Sci 698: 193-203

Rols M-P and Teissié (1990) Electropermeabilization of mammalian cells. Biophys J 58: $1089-1098$ 
Sauer H, Hescheler J, Reis D, Diedershagen H, Niedermeier W and Wartenberg M (1997) DC electrical field-induced c-fos expression and growth stimulation in multicellular prostate cancer spheroids. Br J Cancer 75: 1481-1488

Sersa G, Cemazar M and Miklavcic D (1995) Antitumor effectiveness of electrochemotherapy with cis-diamminedichloroplatinum (II) in mice. Cancer Res 55: 3450-3455

Speelmans G, Staffhorst RW, de Kruijff B and de Wolf FA (1994) Transport studies of doxorubicin in model membranes indicate a difference in passive diffusion across and binding at the outer and inner leaflets of the plasma membrane. Biochemistry 33: 13761-13768

Stanimirovic DB, Wong J, Ball R and Durkin JP (1995) Free radical-induced endothelial membrane disfunction at the site of blood-brain barrier: relationship between lipid peroxidation, $\mathrm{Na}, \mathrm{K}-\mathrm{ATP}$ ase activity, and ${ }^{51} \mathrm{Cr}$ release. Neurochem Res 20: $1417-1427$

Susa N, Ueno S, Furukawa Y and Sugiyama M (1996) Protective effect of vitamin E on chromium (VI)-induced cytotoxicity and lipid peroxidation in primary cultures of rat hepatocytes. Arch Toxicol 71: 20-24

Verstraeten SV, Nogueira LV, Schreier S and Oteiza PI (1997) Effect of trivalent metal ions on phase separation and membrane lipid packing: role in lipid peroxidation. Arch Biochem Biophys 338: 121-127
Wartenberg M, Hescheler J and Sauer H (1997) Electrical fields enhance growth of cancer spheroids by reactive oxygen species and intracellular $\mathrm{Ca}^{2+}$. Am J Physiol 272: R1677-R1683

Wartenberg M, Frey C, Diedershagen H, Ritgen J, Hescheler J and Sauer H (1998a) Development of an intrinsic P-glycoprotein-mediated doxorubicin resistance in quiescent cell layers of large, multicellular prostate tumor spheroids. Int $J$ Cancer 75: 855-863

Wartenberg M, Hescheler J, Acker H, Diedershagen H and Sauer H (1998b) Doxorubicin distribution in multicellular prostate cancer spheroids evaluated by confocal laser scanning microscopy and the 'optical probe technique'. Cytometry 31: 137-145

Weaver JC (1993) Electroporation: a general phenomenon for manipulating cells and tissues. J Cell Biochem 51: 426-435

Wedekind P, Kubitschek U, Heinrich O and Peters R (1996) Line-scanning microphotolysis for diffraction-limited measurements of lateral diffusion. Biophys J 71: 1621-1632 